\title{
Updates on 2016 CNS tumor classification - Where do we stand?
}

\author{
Manish Kolakshyapati ${ }^{1}$, MD, PhD; Tejashwi Shrestha², MD; Anish Man Singh ${ }^{1}$, MS; Rupendra \\ Bahadur Adhikari ${ }^{3}$, MD, PhD; Prabin Shrestha ${ }^{1}, \mathrm{MD}, \mathrm{PhD}$ \\ ${ }^{1}$ Department of Neurosciences, B\&B Hospital, Lalitpur, Nepal \\ ${ }^{2}$ Department of Clinical Neurosciences \& Therapeutics, Graduate School of Biomedical \& Health Sciences, Hiroshima \\ University, Hiroshima, Japan \\ ${ }^{3}$ Department of Neurosurgery, Grande International Hospital, Kathmandu, Nepal
}

\section{Corresponding author}

Manish Kolakshyapati, MD, PhD

email: manish.kola@gmail.com

Received 30 Nov 2018

Accepted 21 Dec 2018

\section{ABSTRACT}

The updated 2016 World Health Organization (WHO) classification of central nervous system (CNS) tumors has incorporated the molecular markers together with histological parameters in defining and diagnosing various tumor entities. This has led to major changes and revision of the classification. A number of new entities are classified based on clinical, diagnostic, and prognostic relevance and those without such relevance have been removed. Major changes are incorporated in diffuse glioma, medulloblastoma, pediatric gliomas, and other embryonal tumors. These new requisites make molecular testing a basic requirement for CNS tumor diagnosis and treatment. New treatment protocols are designed and targeted based on the genetic alteration involved in the tumor entity. The update aims to achieve accurate diagnosis, determine precise prognosis and ensure better patient treatment all through facilitating better categorization of clinical and experimental trials. The multidisciplinary team of clinicians treating such tumor patients in a developing country like Nepal should try to adopt this updated version of the classification so as to improve and upgrade the neuro-oncological services to meet the international standards.

\section{Introduction}

The internationally and widely accepted system of classificationforCNStumorsistheWHOclassification system. The 2007 revision was primarily based on the histological features of the tumor in HE stained sections as observed under the light microscope and ultrastructural characteristics alone ${ }^{1}$. Many studies and researches have explained the genetic basis of tumorigenesis and the role of specific genetic alterations in the origin and progression of tumors ${ }^{2}$. Previously, the molecular alterations were only regarded as to having prognostic value. The 2016 update incorporated the molecular parameters in addition to histology, expanding and restructuring the classification relying more on the molecular markers. This strategy aims to achieve a precise diagnosis and accurate prognosis for better patient management and treatment response. The revised classification of CNS tumors has fused the genetic and histologic characteristics, added some new entities and removed few variants with no diagnostic, clinical or biological relevance with the objective of facilitating the clinical and experimental studies, all directed towards improving the quality of life of brain tumor patients.

\section{Terminologies}

Despite emphasis on the molecular markers, the classification is still structured based on the anatomical site and cells of origin. To standardize the terminologies used for classification, the diagnosis of CNS tumors consists of the histological diagnosis followed by the molecular feature separated by a comma and an adjective (e.g. Glioblastoma, IDHmutant).

For tumors with multiple genetic alterations, the prime alterations should be included in the name itself (e.g. Oligodendroglioma, IDH-mutant and 1p/19q-codeleted). 
For tumors without specific genetic alterations, the term 'wildtype' is used (e.g. Glioblastoma, IDHwildtype).

The term NOS (not otherwise specified) is used in cases where the tumor is not adequately tested for the molecular markers or for the locations where the genetic testing facilities are not available (e.g. Glioblastoma, NOS; Diffuse astrocytoma, NOS). NOS should also be used in rare cases where adequate genetic testing does not reveal any genetic alterations. In short, the term NOS refers to tumors which require further study and/or research.

Grading of CNS tumor is still based on the histological characteristics and are represented by the Roman numerals (e.g. Grade-I, Grade-II, GradeIII and Grade-IV).

\section{Notable changes in 2016 update of CNS tumors}

The significant update in the 2016 classification is the incorporation of the molecular parameters in the diagnosis of a number of tumors with emphasis on the molecular markers. For instance, histologically proven astrocytoma if has IDH mutation and $1 p / 19 q-$ codeletion, is now classified as Oligodendroglioma, IDH-mutant and $1 p / 19 q-$ codeleted. Similarly, tumor that histologically resembles Oligodendroglioma but has IDH mutation and intact $1 p / 19 q$ is diagnosed as Diffuse astrocytoma, IDH-mutant. This has enabled classification of almost all cases of Oligoastrocytoma as either Astrocytoma or Oligodendroglioma with the term Oligoastrocytoma reserved for cases without adequate genetic testing, or rarely those with genetic markers of both astrocytoma and Oligodendroglioma.

Major notable changes include incorporation of molecular markers and restructuring the classification of diffuse glioma, medulloblastoma, embryonal tumors, ependymoma, and pediatric tumors. In addition to this, few newly recognized entities, variants and patterns are added. Variants are the clinically relevant subtypes of entities whereas patterns represent the clinic-pathologically insignificant histological features.

\section{The new additions in the 2016 update are ${ }^{1}$ :}

Entities - Glioblastoma, IDH-mutant and wildtype; Diffuse midline glioma, H3 K27M-mutant; Embryonal tumor with multilayered rosettes, C19MC-altered; Ependymoma, RELA fusion- positive; Diffuse leptomeningeal glioneuronal tumor; Anaplastic PXA

Variants - Epithelioid glioblastoma

Pattern - Glioblastoma with primitive neuronal component; Multinodular and vacuolated pattern of ganglion cell tumor

Othersignificantadditionsincludecombiningsolitary fibrous tumor of dura with hemangiopericytoma as they are considered different spectrum of the same disease with different imaging appearances; brain invasion as a criterion for atypical meningioma.

Similarly, former terms and classifications removed from the new update include: Gliomatosis cerebri; Protoplasmic and fibrillary astrocytoma variant; Cellular ependymoma variant; Primitive neuroectodermal tumor (PNET).

\section{Classification}

The updated 2016 classification of the CNS tumors in tabulated form can be accessed at the following URL - (https://link.springer.com/content/ pdf/10.1007\%2Fs00401-016-1545-1.pdf) ${ }^{3}$. Only the major changes in the disease entities will be discussed in this section.

\section{Diffuse gliomas}

All the infiltrating gliomas, irrespective of the cell of origin, are now grouped together based on the mutation in the driver gene IDH-1 and IDH2 , presence or absence of $1 p / 19 q$-codeletion, ATRX loss and TP53 mutation. This has facilitated in determining the accurate prognosis and better patient management based on the targeted therapies. Figure 1 shows the simplified algorithm for classification of diffuse gliomas.

Diffuse glioma represents the entity where the molecular parameters clearly triumph over the histologicalparameters. Accordingtothenewupdate, the diagnosis of Oligodendroglioma demands testing genetic alterations and demonstration of IDH mutation and codeletion of $1 p / 19 q$. In a setting with negative immunohistochemical staining for IDH mutation, sequencing of IDH-1 132 and IDH2172 is mandatory. In the absence of sequencing capabilities, the histological diagnosis should be made as Oligodendroglioma, NOS. Recently, few researchers have demonstrated the comparable efficacy of PCR-HRM (polymerase chain reactionhigh resolution melt) analysis to genetic sequencing 


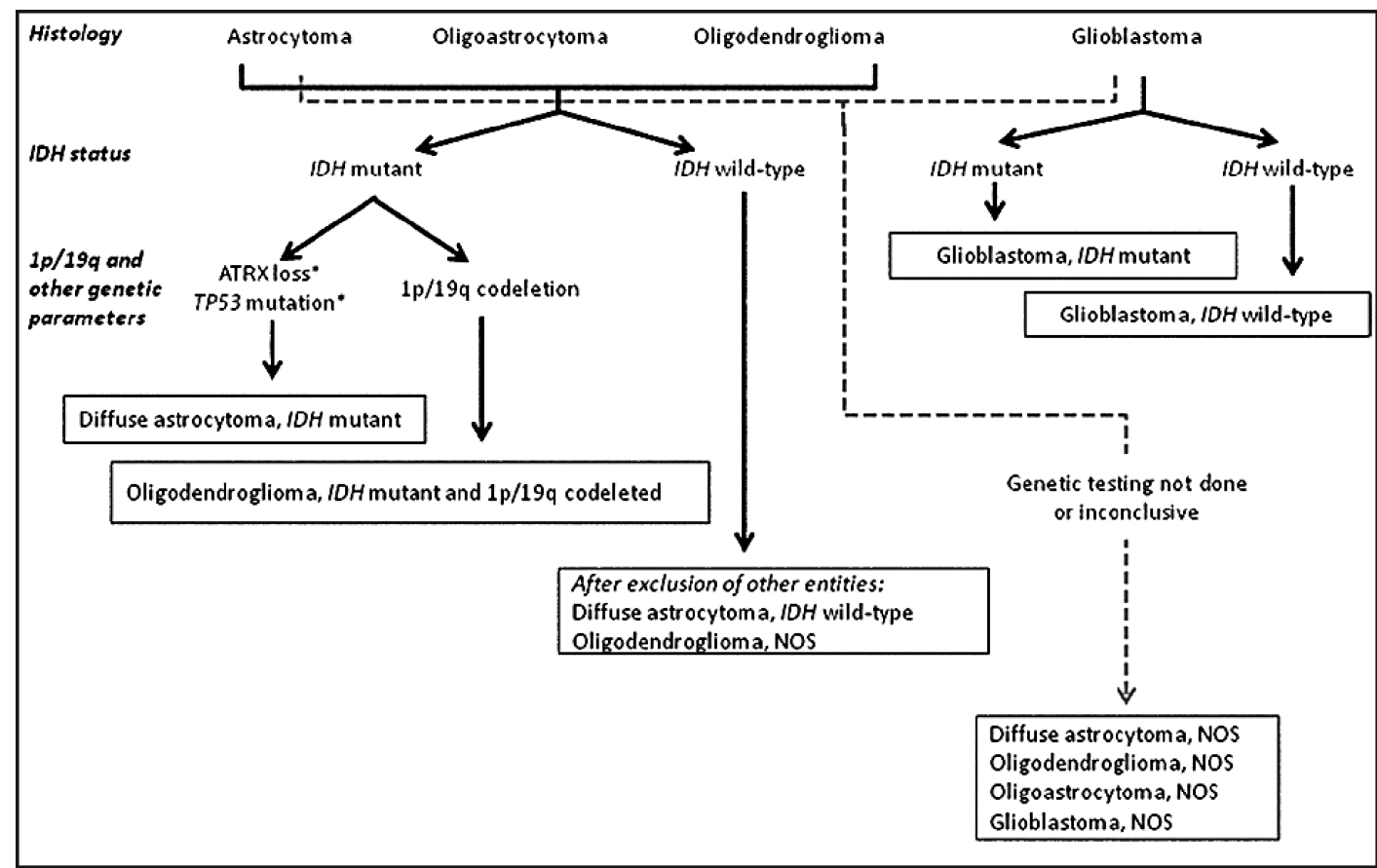

Figure 1: Classification of diffuse gliomas according to updated 2016 classification of CNS tumors based on histological and molecular parameters represented in a simplified algorithm. (Adapted from: Louis DN. (2016) WHO classification of tumors of the central nervous system (IARC))

of IDH1 and IDH2 for rapid analysis of IDH gene mutation ${ }^{4}$. Furth ermore, Oligodendroglioma with $1 p / 19 q$-codeletion has been shown to have better prognosis.

Astrocytoma has also been categorized as Astrocytoma, IDH-mutant, IDH-wildtype and NOS based on driver gene IDH. Further analysis of ATRX and TP53 showed characteristic ATRX loss and TP53 mutation frequently associated with astrocytoma and not with Oligodendroglioma.

Glioblastoma has also been categorized as: Glioblastoma, IDH-wildtype; Glioblastoma, IDHmutant; and Glioblastoma, NOS. Glioblastoma, IDH-wildtype represents almost $90 \%$ of cases and corresponds with clinically defined primary glioblastoma predominantly in patients above 55 years of age. Glioblastoma, IDH-mutant represents $10 \%$ of cases and corresponds with clinically defined secondary glioblastoma preferentially in younger patients. Predominance of IDH-mutation in younger patients suggests the necessity of further evaluation with sequencing for IDH in a setting of negative IDH-immunohistochemistry. Glioblastoma, NOS represents the cases where adequate evaluations have not been or could not be performed. This can simply be represented in the algorithm as shown in Figure 2. Addition of molecular markers to categorize the tumors has further facilitated targeted therapies. Glioblastoma is considered a heterogeneous tumor with multiple genetic alterations and the updated classification has enabled gene-editing with CRISPR technology (still under trial) as a therapeutic modality by better categorization of clinical and experimental trials.

\section{Pediatric diffuse gliomas}

Despite similar histological appearances, pediatric and adult gliomas behave differently and have different prognosis. Previously classified diffuse intrinsic pontine glioma (DIPG) has been newly classified based on the mutational status of H3K27M on histone-3 as diffuse midline glioma, H3K27M-mutant which has diffuse growth pattern and involves the midline structures (thalamus, brain stem and spinal cord) and predominantly occurs in children. This classification is rational for targeted therapies against the mutation responsible for the disease, however, this mutation carries a worse prognosis.

\section{Ependymoma}

A genetically defined variant of ependymoma: Ependymoma, RELA-fusion positive, accounting for majority of supratentorial ependymomas in children characterized by L1CAM expression, has been introduced in the updated classification. 


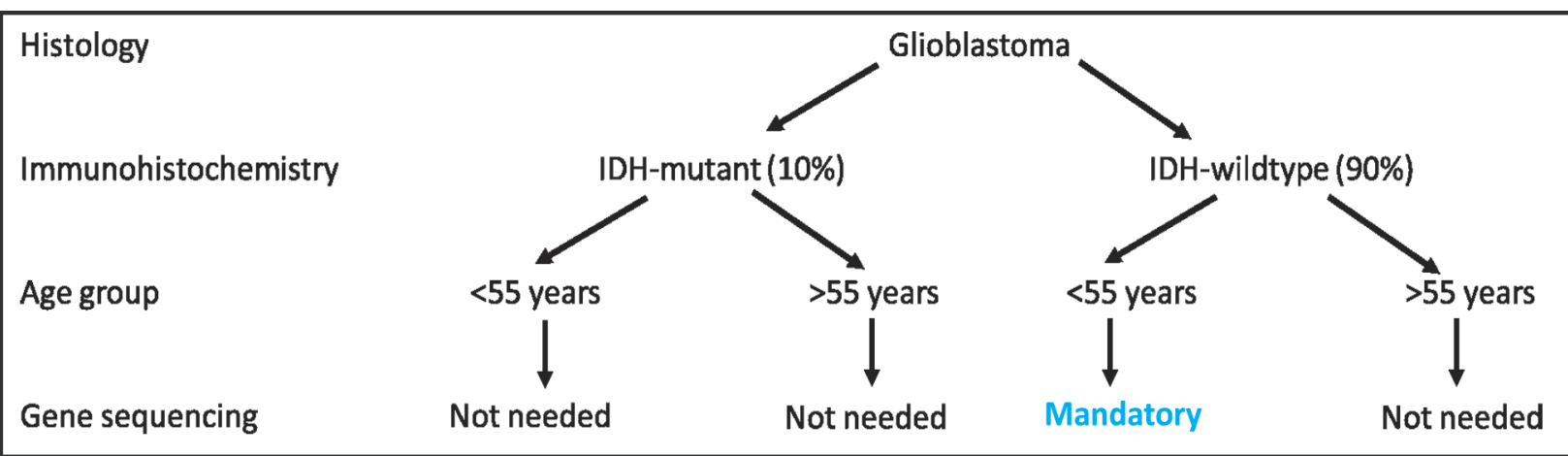

Figure 2: Simple algorithm showing the requirement of gene sequencing for confirming the mutation status of IDH based on age group in glioblastoma patients.

\section{Medulloblastoma}

The updated classification of medulloblastoma represents the most appropriately fused histological and molecular parameters. Medulloblastoma has been genetically defined into four distinct groups with different prognosis: Medulloblastoma, WNTactivated; Medulloblastoma, SHH-activated; and the numerically designated "Group 3"; "Group 4" with the best prognosis in Medulloblastoma, WNT-activated. Histologically, medulloblastomas are categorized as: Medulloblastoma, classic; Medulloblastoma, desmoplastic/nodular; Medulloblastoma with extensive nodularity; Medulloblastoma, large cell/ anaplasticand Medulloblastoma, NOS. The integrated diagnosis incorporates both the histological and molecular parameters and prognosticates based on these parameters.

\section{Summary}

The 2016 update on CNS tumors has made drastic changes in the classification incorporating and emphasizing on the molecular markers over histological parameters. This has paved a way towards the new molecular era in diagnosis and targeted treatment of CNS tumors. The new update aims to achieve accurate diagnosis, determine precise prognosis and ensure better patient treatment in terms of survival, and importantly, quality of life all through facilitating better categorization of clinical and experimental trials.

The developed countries are now moving towards targeted therapies and gene-editing using CRISPR technologyaimedat correcting the geneticalteration involved. Pragmatically, there are a lot of hindrances and challenges in adopting this new classification particularly in developing countries like Nepal. The main challenges in embracing this classification are technological - availability and access to genetic testing and genotyping assays, molecular techniques and basic immunohistochemical stains, and financial strain and bureaucratic red tapes in poor-income countries. A multi-disciplinary team approach to CNS tumors also requires all the physicians involved - Neurosurgeons, Neurologists, Radiologists, Pathologists, Medical/Radiation Oncologists to update their CNS tumor protocols to remain in sync their counterparts. The OPP (out-ofpocket-payment) system further creates hindrances adopting this updated version.

Wider acceptance and practicality of this classification for better treatment protocols and outcomes in brain tumor patients suggests that we should overcome these challenges, sooner rather than later, and upgrade the neuro-oncological services.

\section{References}

1. Louis DN, Ohgaki H, Wiestler OD, Cavenee WK. (2016) WHO classification of tumors of the central nervous system. International Agency for Research on Cancer (IARC), Lyon, France.

2. Louis DN. (2012) The next step in brain tumor classification: "Let us now praise famous men"... or molecules? Acta Neuropathol 124:761-762.

3. Louis DN, Perry A, Reifenberger G, von Deimling A, Figarella-Branger D, Cavenee WK, et al. The 2016 World Health Organization Classification of Tumors of the Central Nervous System: A summary. Acta Neuropathol. 2016;131(6):803-20.

4. Adachi Jl. (2014) Rapid IDH1 Gene Mutation Analysis for Intraoperative Pathological Diagnosis. JSM Neurosurg Spine 2(3): 1028. 